\section{"No dejarse caer en cama": las mujeres y los servicios de salud}

\author{
Women and healthcare services in Mexico: \\ the struggle to avoid becoming "bedridden"
}

Isabel Hernández-Tezoquipa ${ }^{1}$ María de la Luz Arenas-Monreal 1 Rosario Valdez-Santiago ${ }^{1}$

\section{Introducción}

The objective of this study was to identify, describe, and analyze the perception of healthcare services by middle-aged women in five different regions of Mexico. The qualitative analysis was based on 24 in-depth interviews with middleaged women (40-60 years) representing five different regions of the country. The women generally described their health as "constantly poor" but did not allow themselves to become "bedridden". The vast majority of the women postponed going to healthcare services. They considered their health problems insufficiently treated and did not receive enough information for proper self-care. When they did not receive the response they were looking for from healthcare services, women generally sought alternative solutions by regulating their own treatment regimes and self-medication. These experiences provide a strong reference for restructuring key aspects in the current medical model.

Women's Health; Health Services; Middle Aged
En la presente investigación nos enfocamos en la vinculación entre la construcción social de la identidad femenina ${ }^{1}$ y su relación con la percepción de la enfermedad en las mujeres de mediana edad, así como las relaciones de poder que se expresan en la relación médico-paciente, cuando las mujeres en este periodo de vida acuden a los servicios de salud en busca de atención. Retomamos el concepto de género entendido como la construcción cultural simbólica que se hace a partir de los sexos biológicos; se asigna a la persona una manera de ser mujer $\mathrm{u}$ hombre, con formas de comportamiento, de actitudes, destrezas y roles específicos para desenvolverse y ocupar un lugar en el mundo 2 .

La mayoría de los estudios sobre la salud de las mujeres se han centrado particularmente en la etapa reproductiva 1,3. Esta tendencia también se aprecia en los programas de atención a la salud y las políticas públicas, que se centran especialmente en objetivos relacionados con el control de la natalidad y la salud materno-infantil. Dejando de lado otros aspectos de la salud y necesidades de las mujeres que se encuentran en otros momentos del ciclo de vida, como es la mediana edad.

A pesar de que las mujeres de edad mediana constituyen un importante sector de la población, los estudios sobre condiciones de su sa- 
lud y demandas de atención de esta población son prácticamente inexistentes en nuestro país. Algunos estudios muestran que en esta etapa de la vida, las mujeres se preocupan por otros aspectos diferentes a los de salud reproductiva; debido a las modificaciones de su cuerpo, a las experiencias de enfermedad y muerte dentro de los miembros de su familia y el ciclo de vida familiar actual, ya que sus hijos son adultos jóvenes. Estos cambios se conjugan para que su rol de cuidadoras de los otros se modifique y se empiecen a enfocar en su propia salud bajo un concepto más integral, donde lo importante es el balance entre lo espiritual, lo físico y lo emocional 4,5.

Las ciencias sociales han aportado datos significativos que indican que no existe una relación directa entre el sentirse enfermo y acudir al médico. Este fenómeno está mediado por un proceso social en el que interviene la percepción individual, la forma en que se interpretan los síntomas y las características de los sistemas de salud 6. Dentro del gran espectro de variables y condiciones que intervienen en el uso o no de los servicios de salud, se encuentra la relación médico-paciente, la cual juega un papel central en el patrón de utilización 7,8,9,10. Este encuentro social está precedido de supuestos sobre el propósito de la reunión, por la condición de clase, étnica y de género y por el papel de cada uno de los participantes 11.

La persona que demanda la consulta médica lo hace con una historia social implícita. El encuentro social entre el médico y el paciente se da mediante una relación de poder. Para la llamada perspectiva de la economía política, Waitzkin 12 ha señalado las diferentes formas de expresión de la estratificación en la práctica médica. Una de ellas es la información desigual entre médico y paciente.

Otra corriente como Armstrong 13 critica el análisis marxista del poder en la relación médico-paciente como una concepción simplista, al considerar al médico en un ejercicio del poder absoluto sobre el cuerpo de los pacientes. Este autor sugiere que este modelo del poder sólo analiza los elementos manifiestos del poder médico y tiene poca capacidad explicativa para los momentos en los cuales médico y pacientes colaboran para resolver problemas de enfermedad o cuando los pacientes resisten de diferentes formas el poder médico. Las estrategias utilizadas por el paciente dependerán de sus antecedentes, sociales y familiares, nivel de conocimiento, mayor poder social, étnico o de género.
El presente artículo analiza el proceso social implícito entre el "malestar" de las mujeres, entendido como un modo en que las mujeres perciben sus padecimientos y trastornos psíquicos, de una manera que no les permite sentirse saludables pero tampoco enfermas, una ambigua percepción de incomodidad y desasosiego 14 y el acudir a los servicios de salud y las iniciativas que ellas realizan cuando consideran que en los servicios de salud no encuentran respuesta a su malestar.

\section{Método}

Se analizó una base de datos que corresponde a la Encuesta Nacional de Salud (ENSA II) en México. La ENSA II se llevó a cabo en 1994, un componente fundamental de la Encuesta fue la inclusión de un componente cualitativo. La unidad de análisis de este estudio fueron mujeres usuarias y no usuarias de los servicios de salud dentro del rango de edad de 40 y 60 años. El sistema de salud mexicano se encuentra organizado por los servicios de salud de seguridad social, ofrecidos a la población que cuenta con un empleo formal; los servicios de salud, prestados a la población abierta que no cuenta con empleo y los privados. Así la población mexicana atiende su salud a través de los sistemas de atención públicos y privados.

Se agrupó el total de entidades federativas en cinco regiones de estudio: Zona Metropolitana, Centro, Norte, Sudeste y Golfo. En total se realizaron 192 entrevistas a profundidad a siete grupos de población: (a) enfermos que recibieron atención médica, (b) enfermos que no recibieron atención médica, (c) no enfermos que recibieron atención médica (v.g. vacunas, planificación familiar, control de embarazo, detección oportuna de cáncer etc.) y (d) enfermos crónicos. Del total de entrevistas realizadas (192), sólo 24 correspondieron a mujeres de 40 a 60 años de edad.

Estas entrevistas contienen los siguientes ejes temáticos: (1) noción y percepción de salud enfermedad, (2) carrera de la enferma, (3) percepción de los servicios de salud y economía de la salud y (4) hospitalización. Las entrevistas fueron realizadas por personal del área de ciencias sociales con experiencia en la aplicación de este tipo de técnica. El análisis de las 24 entrevistas de este estudio se basó en los tres primeros ejes temáticos, del tercero sólo se consideró la percepción de los servicios de salud. 
Las entrevistas fueron grabadas con la autorización, en todos los casos, de las entrevistadas, se trascribieron y para efectos de codificación y procesamiento de información, se utilizó el programa de cómputo Ethnograph 4.0.

Las categorías analíticas fueron: (1) percepción de las mujeres sobre su malestar, (2) postergación de la salud personal, (3) relación de poder/subordinación y servicios de salud, (4) resistencia de las mujeres al poder médico; éstas se construyeron a partir de los códigos: $a$ sí misma, y ámbito privado para la primer categoría, para la segunda: otros, para la tercera: ámbito público y género y para la cuarta: autoatención, médicina doméstica, medicina tradicional, observación. Tanto los códigos como las categorías se construyeron a partir del campo empírico de la investigación.

\section{Resultados}

De las 24 mujeres entrevistadas, 11 pertenecen al grupo de edad de 40 a 44 años, 5 de 45 a 49, 4 de 50 a 54, 4 de 55 a 59. Respecto al lugar de residencia: 8 mujeres son de la región Sur Mérida y Veracruz, 3 de la región Norte Chihuahua y Saltillo, 2 de la región Centro Aguascalientes y Morelia, y 11 de la región considerada de alta marginación Chilpancingo y Pachuca.

Se presentan los resultados del análisis de las entrevistas realizadas a las mujeres de mediana edad, a partir de las siguientes categorías analíticas: la percepción de las mujeres sobre su malestar; la postergación de la atención de la salud personal; relación de poder/subordinación al solicitar los servicios de salud y la resistencia de las mujeres al poder médico.

La percepción de las mujeres

sobre su malestar

Uno de los apartados centrales de la indagación se relaciona con la percepción y manera en que las mujeres perciben y se expresan respecto a su salud. En este sentido, una respuesta generalizada fue una constante sensación de sentirse enfermas, un malestar generalizado que si bien no las incapacita para llevar a cabo sus actividades diarias, sí está presente como malestar continuo. Estas son algunas de las expresiones en que las mujeres se manifiestan acerca de su percepción de salud: "siento cansancio constante, pienso que en cualquier momento reviento" (Entrevista n. 248); "ya llevo ocho años, rezo a Dios de lo mal que me siento" (Entrevista n. 139); "tengo dolencias por todo el cuerpo" (Entrevista n. 166); "desde niña me he sentido mal, desde los diecisiete años empezó y hasta ahora, de repente pierdo fuerza en el pie y caigo, ayer tres veces me pasó" (Entrevista n. 19).

El malestar de las mujeres se manifiesta por medio de dolores en diferentes partes del cuerpo, decaimiento general y constante. Un sentirse mal continuo que produce una sensación de desventura e imposibilidad de cambio, tanto por las características del mismo como por lo prolongado del padecimiento. Varias de las entrevistas realizadas confirman ese temor: "me siento muy mal pero no me dejo caer en cama” (Entrevista n. 20); “a pesar de sentirme muy mal no estoy tirada, estoy caminando" (Entrevista n. 44).

Las mujeres en este estudio manifiestan respecto a su salud una sensación de sentirse mal constantemente, Burin 14, lo conceptualiza con el término “malestar femenino". Este malestar femenino es identificado por las mujeres cuando se les pide que verbalicen su estado de salud, el cual es muy diferente a lo que registran los servicios que generalmente lo relacionan con aspectos relacionados a salud reproductiva.

Frente a este malestar femenino, una de las reacciones principales de las mujeres es que aunque ellas sienten ese malestar constante, no asumen que su salud se ha alterado y que necesitan ayuda de un profesional para conocer lo que pasa en su cuerpo. Las mujeres conviven con su malestar sin solicitar atención al mismo, es decir, ellas mismas no se declaran enfermas.

\section{La postergación de la salud personal}

Como parte del fenómeno de sentirse mal constantemente y no dejarse caer en cama, las mujeres postergan atender su salud de manera continua. Van al médico cuando se sienten muy mal, es decir, cuando ven amenazado su rol de reproductoras sociales. Las mujeres se sienten enfermas, pero hay un largo proceso de espera entre su sensación de malestar y la búsqueda de ayuda, varios testimonios demuestran estos largos períodos de sentirse enfermas: "tardé ocho días con un óbito" (Entrevista n. 161); "estuve veintidós días con un dolor de muelas hasta tener un dolor terrible por la infección" (Entrevista n. 164); "hasta que convulsione me atendî" (Entrevista n. 53).

Las mujeres y su percepción de salud-enfermedad-atención esta envuelta en un proceso que presenta varias etapas. Puede describirse de esta forma: sentirse mal constantemente, tardar tiempo en declarase enfermas y tardar también en solicitar ayuda en los servicios de salud. El punto central de las mujeres es evitar 
caer en cama, pues se limitaría su función principal como cuidadora de los demás. Estos testimonios ejemplifican lo anterior claramente: "tardo tiempo en atenderme, porque hay muchos a quienes atender" (Entrevista n. 58); "pues yo no sé por qué no hago caso, pues digo, me voy a andar por allá, y ellas van a llegar, y como ahora, pues no barro, ni hago la comida, ellos van a llegar a las dos, y se van en la mañana a las seis y media, y entonces llegan y traen hambre, y quién las va a esperar, pues yo" (Entrevista n. 41).

Estos testimonios ejemplifican el aplazamiento de la atención personal de las mujeres, provocando un constante sentirse mal, pero que les permite cumplir con su rol social de madre-esposa. Diversos estudios muestran que no existe una relación directa entre sentirse enfermo e ir al médico, ya que este fenómeno está mediado por un proceso social en el que interviene la percepción individual y la forma en que el paciente interpreta los síntomas 6 . En el caso de las mujeres está en función del cuidado a los demás. Como lo confirman los resultados de esta investigación, las mujeres comprometen su salud y esperan largos periodos para solicitar atención. No es que no perciban el malestar, ni quieran sobrevivir con él, sino más bien tienen una limitada autonomía y control sobre sus propias necesidades, las que le atañen sólo a ella y su cuerpo. Por otro lado, también pone en evidencia la forma en que las mujeres priorizan sus necesidades en función de las necesidades de los otros, los que están bajo su cargo. Los datos de este estudio son diferentes a los proporcionados en investigaciones realizadas con mujeres de mediana edad en países desarrollados, en las que se refiere que, aunque las mujeres continúan en su rol de cuidadoras, tienen una visión diferente de los riesgos de salud, nuevas oportunidades y otras actitudes para el cuidado de sí mismas 3,4,5.

En general las mujeres de esta investigación perciben que son responsables del cuidado de su familia. Su rol dominante como cuidadora tiene consecuencias en su salud. En este estudio se demuestra la afectación que padecen al acudir tardíamente a los servicios de salud en función del cuidado a los demás. La asignación genérica femenina exige que su rol social sea cumplido de esta forma. Esta asignación social se refleja en la construcción subjetiva de la identidad femenina. En el caso de las mujeres es su función social la que determina lo que realmente pueden hacer por su salud.
Relación de poder/subordinación

\section{y servicios de salud}

La relación entre las mujeres usuarias de los servicios y los profesionales de la salud en el ámbito institucional está caracterizada por el poder que los expertos ejercen sobre ellas. Las mujeres señalan sus necesidades de información y de una explicación de su padecimiento. Esa situación se expresa de varias maneras: "no dijo nada [el médico] o sea que son doctores que no especifican bien lo que tiene uno. Pienso que esos doctores deberían de explicarle a uno por qué fue el motivo de esto [el padecimiento] ¿Por qué se desarrolló? Creo que ya teniendo unos estudios ellos ya deben de ver y más o menos orientar a uno ¿no? Hay doctores que no se expresan bien nada más su recetita. '-Aquí está, se toma esto y esto y lo otro'. De sus estudios, está muy bien, y hasta ahí, 'si siente alguna molestia se vuelve a presentar'. Va uno a presentarse y le dan a uno lo mismo, entonces uno dice para qué cosas voy al seguro" (Entrevista n. 230) (El subrayado es nuestro).

Por otro lado, cuando las mujeres acuden finalmente a los servicios de salud no parecen encontrar una respuesta satisfactoria a su malestar. La relación interpersonal que los prestadores de salud establecen con las mujeres no permite una buena comunicación con el fin de informar sobre la naturaleza de la enfermedad y de los cuidados a realizar.

En esta investigación la relación médicopaciente, como expresión de una relación de poder/subordinación se manifiesta como una relación estresante, las mujeres no reciben un buen trato, existe una falta de información de lo que sucede con su propio cuerpo. El encuentro social entre médicos y las mujeres se da mediante una relación de poder 15 , una de las consecuencias de esta relación es la información desigual entre médico y paciente 11.

El acto de la consulta médica es vivido por las mujeres como una relación de subordinación en el que no sienten ser sujetos participantes, activos e importantes en la atención y cuidado a su salud 2. Esta falta de información y las deficiencias en la relación médico paciente encontradas en este estudio presentan similitud con lo señalado en otras investigaciones realizadas en México 8,9,10. Asimismo, para estas mujeres es muy importante que se integre la exploración física al proceso de diagnóstico, lo cual es semejante a lo señalado en otra investigación que informa de la importancia que las pacientes le asignan a este aspecto ${ }^{9}$. 
Al no encontrar la respuesta que ellas esperan de los servicios de salud, buscan sus propios caminos de acción. En este sentido, no se quedan sin hacer nada, se dan cuenta que al conocer su propio cuerpo pueden explorar por sí mismas cuidados.

\section{- Autodiagnóstico, auto descubrimiento de los cuidados}

Las mujeres al observar sus malestares de salud, confunden los síntomas, no tienen ni reciben información adecuada. Debido a la falta de información por parte de los servicios de salud, las mujeres indagan acerca de qué cuidados deben procurarse para sobrellevar su malestar. Ellas, también descubren los cuidados de salud que les benefician, por ejemplo, en caso de enfermedades crónicas el tipo de alimentación que les favorece. Un testimonio afirma: "entonces teniendo diabetes tiene uno que alimentarse, pero no sabía que yo tenía que alimentarme bien. Ahora que ya pasaron los años, lo sabe uno" (Entrevista n. 74).

Las mujeres por sí mismas van descubriendo qué cuidados les benefician - o no -, ya sea por observación o por ensayo y error, es un conjunto de conocimientos que ellas van acumulando. El cuidado de la salud de las mujeres depende más de la experiencia adquirida por sí mismas que por la relación que establecen con los profesionales de la salud que las atienden en los servicios.

\section{- Autorregulación de tratamientos y medicación}

La utilización de los propios saberes y conocimientos se aplica también al uso de medicamentos y tratamientos. Ellas descubren por su propia experiencia lo que les es nocivo y lo que les ayuda a cuidar su salud. Por ejemplo, un testimonio es el siguiente: "pero yo como al año de que me empecé a vacunar ya me sentía bien, ya no me daba esa alergia, no me daba ese catarro, pero no oía bien. Me dejé de vacunar como dos o tres meses para ver si era eso; entonces sí me di cuenta y el doctor también se dio cuenta de que era eso, entonces por lo mismo ya no me seguí vacunando. Porque vacunas, yo decidí que ya no, yo decidí" (Entrevista n. 53).

La atención recibida en los servicios genera insatisfacción en las mujeres, dando lugar a que realicen su propia búsqueda de cuidadocuración y un proceso de resistencia 13 . Esta resistencia se expresa mediante iniciativas que las mujeres realizan para el cuidado de su salud. Lo anterior se hizo evidente en este estudio, apreciándose que la relación de poder entre el médico y la mujer-usuaria, no es unidireccional, sino que las mujeres como sujetos sociales responden al poder médico mediante la resistencia.

No sólo las mujeres de México detectan este tipo de problemas cuando ellas acuden a los servicios de salud, sino también en países desarrollados, donde las mujeres desean que los servicios de salud a través de sus profesionales se les trate, provean información y brinden una atención con calidad 16. Esto significa que la reflexión se debe centrar en el modelo de atención a la salud que debe escuchar la experiencia, las voces y las necesidades de las mujeres.

\section{Conclusiones}

Los resultados de esta investigación contribuyen a mejorar el conocimiento sobre el camino que recorren las mujeres desde la percepción de su estado de salud, la respuesta que reciben de los servicios de salud y las acciones que realizan ante lo que consideran falta de interés por parte de este sector para resolver sus problemas. En especial en las mujeres de mediana edad de México, se observa en ellas que en esta etapa de su vida no tienen autonomía sobre su propias necesidades, continúan priorizando las necesidades de los otros y postergando la atención de su salud.

De los servicios de salud, las mujeres desean que haya información y comunicación entre ellas y los profesionales de la salud que las atienden, situación que se observa no sólo en México, sino también en países desarrollados 16 .

Desde la época prehispánica la función social de las mujeres era el cuidado de los demás "la madre de familia tiene hijos, los amamanta. Su corazón es bueno, vigilante, diligente, cava la tierra, tiene ánimo, vigila con sus manos y su corazón se afana. Educa a sus hijos, se ocupa de todos, a todos atiende, cuida de los más pequeños. A todos sirve, se afana por todos, nada descuida, conserva lo que tiene, no reposa" (Códice Matritense de la Real Academia) 17 (p. 18). Después de quinientos años, la sociedad continúa esperando lo mismo de las mujeres. Lo anterior sugiere un cambio estructural social, en el que las mujeres sean las constructoras y cuidadoras para sí mismas de sus propias vidas.

La aportación de este manuscrito reside en que ofrece una visión integral del cuidado-atención de la salud de las mujeres: la percepción de las mujeres sobre su malestar; la posterga- 
ción de la atención de la salud personal; la percepción que ellas tienes de los servicios de salud y la resistencia al poder médico.

Una limitación de este estudio es que la base de datos secundarios de tipo cualitativo que se utilizó para este artículo omitió registrar datos sociodemográficos como estado conyugal, número de hijos entre otros.

\section{Resumen}

El objetivo del presente trabajo fue identificar, describir y analizar el ciclo de percepción-atención de salud de las mujeres de mediana edad de cinco regiones del país. Se realizó un análisis cualitativo de 24 entrevistas a profundidad, aplicadas a mujeres de mediana edad (40 a 60 años), procedentes de cinco regiones de México. Los hallazgos de la investigación muestran que la percepción de salud de las mujeres se caracteriza por "sentirse constantemente mal", sin embargo, no se "dejan caer en cama". La búsqueda de atención a la salud en todos los casos es tardía. Las consideran que su malestar no es suficientemente atendido, ni reciben la información necesaria para su cuidado. Al no encontrar la respuesta que ellas esperan de los servicios de salud, buscan sus propios caminos como la autorregulación de tratamientos y la automedicación. La experiencia de las mujeres, es un punto de referencia para conseguir que se reformulen algunos aspectos del modelo médico existente.

Salud de las Mujeres; Servicios de Salud; Mediana Edad

\section{Colaboradores}

I. Hernández-Tezoquipa ha contribuido en el diseño y concepción general del trabajo. M. L. Arenas-Monreal participó en la descripción de los resultados. R. Valdez-Santiago realizó el análisis y marco teórico. 


\section{Referencias}

1. Szasz I. Género y salud. Propuesta para el análisis de una relación compleja. In: Brofman M, Castro $\mathrm{R}$, editors. Salud, cambio social y política. Perspectivas desde América Latina. México DC: EDAMEX; 1999. p. 109-22.

2. Sayavedra Herrerías G, Flores Hernández E. Ser mujer: ¿Un riesgo para la salud? Del malestar y enfermar, al poderío y la salud. México DC: Red de mujeres; 1997.

3. Villela M, Hilfinger D, Keiko A, Partezani RA, Spano AM, Almeida AM. Mulher e saúde: suas preocupações. Rev Paul Enfermagem 1991; 10: 121-7.

4. Bruenjes S. Orchestrating health: middle-aged women's process of living health. Holist Nurs Pract 1994; 8:22-32.

5. Devine CM, Olson CM. Women's perceptions about the way social roles promote or constrain personal nutrition care. Women Health 1992; 19:79-95.

6. De la Cuesta-Benjumea C. Las mujeres y el manejo de un síntoma de la valoración a la selección. Salud Pública Méx 1999; 41:124-9.

7. Donabedian A. The quality of care. How can it be assessed? JAMA 1988; 260:1743-8.

8. Bronfman M, Castro R, Oviedo J, Zúñiga E, Miranda C. Del "cuánto" al "por qué": la utilización de los servicios de salud desde la perspectiva de los usuarios. Salud Pública Méx 1997; 39:442-50.

9. Cuevas ML, Ávila I, Oiliver J, Sumano M, Palomares G, Garduño J. Relación médico y paciente en la consulta externa de unidades de primer nivel de atención médica. Salud Pública Méx 1991; 33:576-84
10. Ramírez T, Najera P, Nigenda G. Percepción de la calidad de la atención de los servicios de salud en México: perspectiva de los usuarios. Salud Pública Méx 1998; 40:3-12.

11. Tood DA. The patient's view. Intimate adversaries. Cultural conflict between doctors and women patients. Pennsylvania: University of Pennsylvania Press; 1989.

12. Waitzkin $\mathrm{H}$. The politics of medical encounters: how patient's and doctors deal with social problems. Connecticut: Yale University Press; 1991.

13. Armstrong D. The patient's view. Soc Sci Med 1984; 18:737-44.

14. Burin M. Poder, amor y sexualidad en la construcción de la subjetividad. Buenos Aires: Paidós; 1998.

15. Lupton D. Medicine as culture. London: Sage Publications; 1996.

16. Taylor D, Doler C. Toward a women-centered health care system: women's experiences, women's voices, women's needs. Health Care Women Int 1997; 18:407-22.

17. León-Portilla M. Cihuayotl iixco ca: la feminidad luce en su rostro. Arqueología Mexicana 1998; 5:14-9.

Recibido el 05/Abr/2004

Versión final presentada el 04/Ene/2005

Aprobado el 14/Mar/2005 